\title{
OTIMIZAÇÃO DOS CUIDADOS INTENSIVOS NA ASSISTÊNCIA AO PACIENTE COM COVID-19
}

Josefine Busanello ${ }^{1}$

Sabrina Guterres da Silva Galetto ${ }^{2}$

Jenifer Harter ${ }^{1}$

Raquel Potter Garcia ${ }^{1}$

\author{
https://orcid.org/0000-0002-9950-951 \\ https://orcid.org/0000-0002-9130-4290 \\ https://orcid.org/0000-0002-9130-4290 \\ https://orcid.org/0000-0002-5503-7981
}

Objetivo: Refletir acerca das estratégias para a otimização dos cuidados intensivos na assistência ao paciente com COVID-19. Método: Trata-se de um estudo teórico-reflexivo, que resgata as considerações atualizadas acerca da gestão do cuidado intensivo no contexto da pandemia. Resultados: $O$ conceito de otimização do cuidado, aplicado ao contexto de pandemia da COVID-19, pode ser definido como o conjunto de ações ao atendimento das necessidades humanas básicas e a operacionalização do suporte avançado, com vistas à segurança do paciente e à minimização dos riscos relativos à exposição biológica dos profissionais. Nessa perspectiva, é necessário articular segmentos essenciais da assistência: suporte para os profissionais; organização do ambiente; e adaptação das rotinas de cuidado. Conclusão: A otimização do cuidado ao paciente com COVID-19 requer reorganização das unidades de terapia intensiva, treinamentos, provisão de equipamentos de proteção individual e atenção à saúde ocupacional. O espaço físico deve permitir divisão e classificação das áreas, idealmente com leitos de isolamento. Medidas de controle da dispersão do vírus devem ser adotadas e rotinas de cuidado adaptadas.

Descritores: Unidades de Terapia Intensiva; Infecções por Coronavirus; Cuidados de Enfermagem; Cuidados Críticos; Enfermagem.

\section{OPTIMIZATION OF INTENSIVE CARE IN ASSISTENCE FOR THE PATIENT WITH COVID-19}

Objective: Reflect on strategies for the optimization of intensive care in patient care with COVID-19. Method: This is a theoretical-reflective study, which retrieves updated considerations about the management of intensive care in the context of the pandemic. Results: The concept of care optimization, applied to the COVID-19 pandemic context, can be defined as the set of actions to meet basic human needs and operationalize advanced support, with a view to patient safety and minimizing risks related to the biological exposure of professionals. In this perspective, it is necessary to articulate essential segments of assistance: support for professionals; organization of the environment; and adaptation of care routines. Conclusion: Optimizing patient care with COVID-19 requires reorganization of intensive care units, training, provision of personal protective equipment and attention to occupational health. The physical space should allow division and classification of areas, ideally with isolation beds. Measures to control the spread of the virus should be adopted and care routines adapted.

Descriptors: Intensive Care Units; Coronavirus infections; Nursing care; Critical Care; Nursing.

\section{OPTIMIZACIÓN DE LOS CUIDADOS INTENSIVOS EN LAATENCIÓN PACIENTE CON COVID-19}

Objetivo: Reflexionar sobre estrategias para la optimización de los cuidados intensivos en la atención al paciente con COVID-19. Metodo: Este es un estudio teórico-reflexivo, que recupera consideraciones actualizadas sobre el manejo de cuidados intensivos en el contexto de la pandemia. Resultados: El concepto de optimización de la atención, aplicado al contexto de la pandemia de COVID-19, se puede definir como el conjunto de acciones para satisfacer las necesidades humanas básicas y operacionalizar el soporte avanzado, con miras a la seguridad del paciente y minimizar riesgos relacionados con la exposición biológica de profesionales. En esta perspectiva, es necesario articular segmentos esenciales de asistencia: apoyo a profesionales; organización del medio ambiente; y adaptación de rutinas asistenciales. Conclusión: La optimización de la atención al paciente con COVID-19 requiere la reorganización de las unidades de cuidados intensivos, capacitación, provisión de equipos de protección personal y atención a la salud ocupacional. El espacio físico debe permitir la división y clasificación de áreas, idealmente con camas de aislamiento. Se deben adoptar medidas para controlar la propagación del virus y adaptar las rutinas de atención.

Descriptores: Unidades de Cuidados Intensivos; Infecciones por coronavirus; Cuidado de enfermera; Cuidado crítico; Enfermería.

IUniversidade Federal do Estado do Rio de Janeiro.

${ }^{2}$ Instituto Nacional de Câncer. Universidade Federal do Estado do Rio de Janeiro.

Autor Correspondente: Camila Lopes Liandro - Email: liandro1989@gmail.com

Recebido: 17/06/2019 - Aceito: 09/11/2019 


\section{INTRODUÇÃO}

As Unidades de Terapia Intensiva (UTI) se constituíram em cenários de destaque frente à pandemia, considerando a necessidade de suporte tecnológico e de cuidados avançados aos pacientes com Corona Virus Disease (COVID-19)1. A infecção por Severe Acute Respiratory Syndrome Coronavirus 2 (SARS-CoVv-2) pode desencadear condições clínicas graves, que acometem todas as funções vitais, não se restringindo apenas ao sistema respiratório. Aproximadamente cinco por cento das infecções comprovadas necessitam cuidados críticos em UTI², com equipamentos tecnológicos específicos e profissionais habilitados para realizar os cuidados com segurança.

Nesse contexto, são inúmeros os desafios para os trabalhadores da saúde ${ }^{3}$, especialmente aqueles que mantêm contato contínuo com o paciente no periodo de tratamento intensivo. Aos enfermeiros é exigida a capacidade de inovação, de tomada de decisões e de liderança para criar condições mais favoráveis à implementação dos cuidados na assistência ao paciente com COVID-194-5. Nesse sentido, objetiva-se refletir acerca das estratégias para a otimização dos cuidados intensivos na assistência ao paciente com COVID-19.

\section{DESENVOLVIMENTO}

O conceito de otimização do cuidado, aplicado ao contexto da pandemia de COVID-19, pode ser definido como o conjunto de ações direcionadas ao atendimento das necessidades humanas básicas e a operacionalização do suporte avançado, com vistas à segurança do paciente e a minimização dos riscos relativos à exposição biológica dos profissionais ${ }^{6}$. Nessa perspectiva, o presente estudo apresenta uma reflexão com base nas seguintes categorias: Suporte para os profissionais; Organização do ambiente de trabalho; e Adaptação das rotinas de cuidado.

\section{SUPORTE PARA OS PROFISSIONAIS}

Para garantir a força de trabalho qualificada e suficiente para atender a demanda pandêmica é necessário estabelecer estratégias de apoio para os profissionais da saúde. A complexidade clínica da COVID-19 potencializou a importância de capacitações, por meio de simulações práticas sobre biossegurança e protocolos de tratamento ${ }^{6}$. Além disso, o monitoramento do desgaste emocional e físico vivenciado pelos profissionais é essencial frente à magnitude dessa experiência, que se intensifica na UTI pelo quantitativo de pacientes graves de várias faixas etárias, o dinamismo do cuidado e a alta taxa de letali- dade. Condição agravada pela precariedade de serviços de suporte e apoio para gerenciar depressão, ansiedade, medo e sofrimento psicológico advindos da vivência deste contexto.

Destaca-se a vigilância à saúde do trabalhador, com ênfase nas ações para a avaliação diária das condições de saúde, incluso sinais e sintomas da COVID-19, bem como outras doenças relacionada ao trabalho ou ocupacionais a nível biológico e psicossocial. O acompanhamento dos profissionais é apontado como uma das ações mais efetivas para a manutenção dos trabalhadores da saúde no enfrentamento da pandemia ${ }^{7}$, já que estes são fundamentais para o pleno funcionamento dos serviços de saúde.

Também podem ser instituidas estratégias para a motivação dos profissionais, com a expectativa de ofertar momentos de prazer, superação das dificuldades do cotidiano e, assim, evitar absenteísmos e demissões. Estigmatização social por trabalhar com pacientes com COVID-19, sobrecarga laboral e medo de se contaminar ou contaminar seus familiares, podem afetar potencialmente a saúde mental destes profissionais da saúde ${ }^{8}$.

Experiências vivenciadas em países que já superaram a fase aguda da pandemia destacam a importância de assegurar momentos para descanso e alimentação durante o turno de trabalho, com espaços seguros para retirada dos EPI. O uso ininterrupto desses equipamentos desencadeia desconforto e lesões por pressão na região facial, especialmente pelas máscaras e face shilds ${ }^{9}$. Ademais, a identidade dos profissionais é velada pelos EPI prejudicando a interação e comunicação entre profissionais e pacientes. Para minimizar esses efeitos, iniciativas para resgatar a humanização da assistência à saúde e superar o distanciamento estabelecido, têm sido utilizadas, como por exemplo, a criação de crachás "humanizados", com fotos que destacam o rosto e o nome dos profissionais.

Em contrapartida, há preocupação acerca da escassez e/ou inadequação dos EPI, somada a exposição de profissionais dos grupos de risco que não obtiveram respaldo para o afastamento das atividades de trabalho. No Brasil, mais de 18 mil profissionais de Enfermagem já foram contaminados pelo novo coronavírus e mais de 200 já perderam a vida em decorrência da COVID-19. Esse contexto demonstra a necessidade urgente de tomada de decisão pelos órgãos competentes para garantir a proteção e suporte a estes profissionais ${ }^{11}$, evitando mais prejuizos para uma das principais forças de trabalho no enfrentamento da pandemia. 


\section{ORGANIZAÇÃO DO AMBIENTE DE TRABALHO}

Experiências internacionais acerca da organização do ambiente da UTI destinada a pacientes com COVID-19 orientam para a divisão de áreas que permitam a concentração e segregação de equipamentos e profissionais, a partir da classificação de risco biológico ${ }^{5,10}$. Essa perspectiva resgata a importância histórica da Enfermagem frente às principais crises sanitárias da humanidade. Florence Nightingale, a partir da Teoria Ambientalista, comprovou que a higienização das mãos, o isolamento social, a ventilação dos ambientes, e a classificação dos pacientes para a setorização das áreas de cuidado, já eram medidas efetivas para combater doenças e outras epidemias há 200 anos.

Transpondo o conceito de Florence para a realidade tecnológica atual, idealmente, os leitos destinados aos pacientes com COVID-19 devem contar com pressão negativa ${ }^{5}$, para fins de controle da dispersão de microorganismos no ar e superfícies. No Brasil, esse sistema não faz parte da maioria das UTI, e a precariedade estrutural dessas ficou mais evidente a partir da pandemia. A falta de leitos também é um problema que antecede a pandemia, e também reflete as dificuldades de efetivação da saúde preventiva e promocional no âmbito da atenção básica.

De qualquer modo, fez-se necessária a ampliação emergencial do número de leitos de UTI, muitos em hospitais de campanha, estabelecendo a improvisação de leitos para pacientes graves com COVID-19. Por isso, a maioria das unidades sem espaço e condições físicas para isolamento privativo do paciente, assumiram configuração de coorte, por similaridade de caso ${ }^{5}$. Essa configuração não vai ao encontro de uma assistência de excelência que proporcione segurança para todos os envolvidos no processo, porém é o cenário possível diante da conjuntura de saúde estabelecida.

Um estudo realizado em um hospital da China evidenciou a distribuição do SARS-CoV-2 em superfícies, tais como mouses, lixeiras e corrimãos de leitos da UTI. A contaminação do piso foi confirmada em $70 \%$ das amostras coletadas. No ar, o vírus foi amplamente detectado, sendo dispersado por, aproximadamente, quatro metros dos pacientes. A principal conclusão do referido estudo é que o SARS-CoV-2 não pode ser isolado, contudo as barreiras ambientais podem auxiliar no contingenciamento do patógeno e controle de risco ${ }^{12}$. Assim, a alta transmissibilidade do SARS-CoV-2 impôs a necessidade de medidas para o controle da aerolização durante os procedimentos invasivos das vias aéreas que, até a pandemia, eram subestimadas mesmo diante da incidência de outras doenças infectocontagiosas, como a tuberculose ${ }^{12}$.

A pandemia não resgatou somente cuidados básicos do cotidiano dos serviços de saúde, como a lavagem das mãos e o uso dos EPI, mas fortaleceu a importância do aparato tecnológico na estruturação dos ambientes de cuidado intensivo. Monitores, microfones e câmeras ligadas à central de monitorização, são essenciais para melhorar a comunicação entre os profissionais da equipe ${ }^{10} \mathrm{e}$ os pacientes com COVID-19 em isolamento.

\section{ADAPTAÇÃO DAS ROTINAS DE CUIDADO}

A COVID-19 é uma doença nova, com um curso clínico incompleto, que exige a constante adaptação das rotinas de cuidado intensivo ${ }^{1}$. Nesse sentido, os fluxos ou fluxogramas têm sido uma das alternativas para a comunicação clara e objetiva, consulta rápida e visualização disponivel das ações pertinentes para a assistência, conforme a consolidação do conhecimento na área.

No que tange aos aspectos clínicos, além dos sinais vitais, devem ser avaliados o tempo de enchimento capilar, aspecto e temperatura da pele, valores gasométricos, controle da fluidoterapia e do equilibrio hidroeletrolítico, os quais permitem determinar agravos refratários ao suporte ventilatório e hemodinâmico ${ }^{13}$. A maioria destes cuidados ficam sob responsabilidade dos profissionais da Enfermagem. Nesse sentido, é oportuno refletir sobre a importância da gestão dos cuidados, liderada pelo enfermeiro, e que visa a definição das ações prioritárias no planejamento do cuidado, incluso a adaptação das rotinas da UTI.

Especialmente, sobre as recomendações para o gerenciamento das vias aéreas e o suporte ventilatório, são sugestivas à intubação precoce, com sequência rápida e ventilação protetora ${ }^{14}$. Essa situação se caracteriza de maneira diferente da conduta clínica habitual, o que fomentou a necessidade de adaptação dos profissionais e a própria quebra de paradigmas dentro do cenário de terapia intensiva. Usualmente os meios invasivos são postergados por meio da rotina de instituir métodos ventilatórios não invasivos para reverter insuficiência respiratória aguda de outra causa.

Além disso, as posições prona e autoprona são apresentadas como prática exitosa no tratamento dos pacientes com COVID-19, por aumentar a complacência pulmonar ${ }^{13}$. Todavia, exigem adaptações da rotina de higiene, nutrição, posicionamento e prevenção de lesões por pressão, a fim de evitar eventos adversos. Particularmente, essa estratégia para melhorar a função respi- 
ratória também nos faz refletir sobre a magnitude de cuidados imposta pela complexidade clínica da COVID-19. É necessário planejamento multiprofissional para analisar os riscos e benefícios, além da garantia de segurança durante a própria manobra.

A adaptação para a técnica de banho seco e o controle das eliminações são consideradas medidas necessárias para evitar dispersão do SARS-COv-2 no ambiente $e^{2,15}$. Frente a esse aspecto, cabe resgatar a reflexão que acompanha o desenvolvimento da Ciência da Enfermagem, historicamente vinculada a prática de ações de menor valor, tais como a higiene. Na conjuntura da pandemia, aos cuidados que são de responsabilidade exclusiva da Enfermagem, foram atribuídas percepções de complexidade, relevância clínica e contato contínuo e direto com o paciente COVID-19. Cenário oportuno para o resgate da valorização da Enfermagem enquanto prática social e profissão ativa no processo de produção de saúde.

Estratégias para o envolvimento da família são importantes, face a suspensão das visitas aos pacientes com COVID-19. Para minimizar as preocupações e gerenciar as expectativas ${ }^{5}$, é necessário estabelecer novas formas de comunicação entre o paciente, a família e os profissionais da equipe, as quais podem incluir o uso das tecnologias da informação. Nesse sentido, um dos maiores desafios para os profissionais é a comunicação de más notícias de forma não presencial.

A pandemia da COVID-19 rompeu com os componentes básicos da comunicação, ferramenta tão essencial para o trabalho dos profissionais da saúde. A terminalidade, a morte e o luto foram afetados por perdas em massa, e dificuldades para a realização de rituais de despedida. Assim, os profissionais precisam buscar alternativas respeitosas para ritualização dos processos vividos, a fim de ressignificar as perdas e enfrentar desafios durante e após a vigência da pandemia. Tendo ainda os profissionais que lidarem com o processo de frustração constante frente a esta perda massiva de pacientes ${ }^{16}$

\section{LIMITAÇÕES DO ESTUDO}

As reflexões apresentadas podem conter potencial viés, especialmente no que se refere às experiências de enfrentamento à pandemia, as características epidemiológicas e o padrão institucional hospitalar dos contextos aos quais os autores estão vinculados.

\section{Contribuição do Estudo para a Prática}

As contribuições se pautam, sobretudo no suporte para os profissionais de Enfermagem, na organização do ambiente de trabalho e na adaptação das rotinas de cuidado. Destaca-se a incipiência de estudos e produções científicas sobre o tema em periódicos nacionais, e a emergência de subsidios para o enfrentamento da pandemia, em especial na UTI, ambiente no qual o paciente permanece internado por um período prolongado e os profissionais são expostos a risco biológico elevado constantemente.

\section{CONSIDERAÇÕES FINAIS}

A otimização do cuidado aos pacientes com COVID-19 nas UTI implica em uma reorganização destas unidades, mediante a qualificação dos profissionais, provisão de EPI específicos e atenção à saúde dos trabalhadores. O espaço físico deve ser estruturado de forma que permita divisão e classificação da unidade em áreas específicas, idealmente compostos por leitos de isolamento com pressão negativa. Medidas de segurança para o controle da dispersão do vírus no ambiente devem ser adotadas, pois alguns procedimentos invasivos das vias aéreas geram aerolização. As rotinas de cuidado também precisam ser adaptadas, com base nas evidências científicas disponíveis, no fortalecimento e adaptação de formas de comunicação e na sistematização da assistência.

A reflexão sobre as estratégias para a otimização dos cuidados intensivos na assistência ao paciente com COVID-19, demonstra que inúmeras adequações são necessárias junto ao contexto das instituições de saúde, exigindo conhecimento, segurança e flexibilidade por parte dos gestores para ultrapassar o período vivenciado. Apesar de todas as dificuldades, é possível que, por meio da otimização de cuidados, muitos serviços de saúde se fortaleçam para uma assistência de maior excelência no pós-pandemia.

Contribuições dos Autores: Josefine Busanello: a) concepção e/ou desenho do estudo; b) análise e interpretação dos dados; c) redação e/ou revisão crítica do manuscrito; d) aprovação da versão final a ser publicada; Sabrina Guterres da Silva Galetto: b) análise e interpretação dos dados; c) redação e/ou revisão crítica do manuscrito; d) aprovação da versão final a ser publicada; Jenifer Harter: b) análise e interpretação dos dados; c) redação e/ou revisão crítica do manuscrito; d) aprovação da versão final a ser publicada; Raquel Potter Garcia: b) análise e interpretação dos dados; c) redação e/ou revisão crítica do manuscrito; d) aprovação da versão final a ser publicada. 


\section{REFERÊNCIAS}

1. Murthy S, Gomersall CD, Webb S. Care for Critically Ill Patients With COVID-19. JAMA [Internet]. 2020 [cited 2020 Mai 20]; 323(15):1499-1500. Available from: https://jamanetwork.com/journals/jama/ fullarticle/2762996.

2. Wu Z, McGoogan JM. Characteristics of and Important Lessons From the Coronavirus Disease 2019 (COVID-19) Outbreak in China. JAMA [Internet]. 2020 [cited 2020 Mai 20]; 323(13):1239-1242. Available from: https://jamanetwork.com/journals/jama/ fullarticle/2762130.

3. Jackson D, Bradburry-Jones C, Baptiste D, Gelling L, Morin K, Neville S, et al. Life in the pandemic: some reflections on nursing in the contexto of $\mathrm{CO}$ VID-19. J Clin Nurs [Internet]. 2020 [cited 2020 Mai 20]; 29:2041-2043. Available from: https://onlinelibrary.wiley.com/doi/epdf/10.11l1/jocn.15257.

4. Miranda FMA, Santana LL, Pizzolato AC, Saquis LMM. Condições de trabalho e o impacto na saúde dos profissionais de Enfermagem frente a COVID-19. Cogitare Enferm [Internet]. 2020 [cited 2020 Mai 20]; 25:e72702. Available from: https://revistas.ufpr. br/cogitare/article/view/72702/pdf

5. Goh JK, Wong J, Tien C, Chie J, Yi Ng J, Wen SD, et al. Preparing your intensive care unit for the COVID-19 pandemic: practical considerations and strategies. Crit Care [Internet]. 2020 [cited 2020 Mai 20]; 24(25):02-12. Available from: https:// ccforum.biomedcentral.com/track/pdf/10.1186/ s13054-020-02916-4.

6. Editorial. COVID-19: protecting health-care workers. Lancet [Internet]. 2020 [cited 2020 Mai 20]; 395:922. Available from: https://doi.org/10.1016/ S0140-6736(20)30644-9.

7. Liew MF, Siow WT, MacLaren G, See KC. Preparing for COVID-19: early experience from an intensive care unit in Singapore. Critical Care [Internet]. 2020 [cited 2020 Mai 20];24(83):01-03. Available from:https://ccforum.biomedcentral.com/track/ pdf/10.1186/s13054-020-2814-x.

8. Petzold MB, Plag J, Ströhle A. Dealing with psychological distress by healthcare professionals during the COVID-19 pandemia. Nervenarzt [Internet]. 2020 [cited 2020 Mai 20];91(5):01-05. Available from: https://www.ncbi.nlm.nih.gov/pmc/articles/ PMC7100457/.

9. Lam UN, Md Mydin Siddik NSF, Mohd Yussof SJ, Ibrahim S. N95 respirator associated pressure ulcer amongst COVID-19 health care workers. Int Wound J [Internet]. 2020 [cited 2020 Mai 20]; 10:01-03. Available from: https://onlinelibrary.wiley.com/doi/ full/10.11ll/iwj.13398.

10. Vargas M, Marcos G, Simone S, Servilho G. Logistic and organizational aspects of a dedicated intensive care unit for COVID-19 patients. Critical Care [Internet]. 2020 [cited 2020 Mai 20]; 24(237):01-03. Available from: https://doi.org/10.1186/s13054020-02955-x

11. Santos VC, Persegona MFM, Souza EF, Almeida WC, Filete M, Silva MCN. Comitê Gestor de Crise do Coronavirus no âmbito do COFEN. Enferm. Foco [Internet]. 2020 [cited $2020 \mathrm{Jul}$ 24]; 10(6):6-10. Available from: http://revista.cofen.gov.br/index.php/ enfermagem/article/view/4213/730.

12. Guo ZD, Wang ZY, Zhang SF, Li X, Li L, Li C, et al. Aerosol and surface distribution of severe acute respiratory syndrome coronavirus 2 in hospital wards, Wuhan, China, 2020. Emerg Infect Dis [Internet] 2020 [cited 2020 Mai 20];26(7): 1583-1591. Available from: https://doi.org/10.3201/eid2607.200885.

13. Bein B, Bachmann M, Hugget S, Wegermann P. SARS-CoV-2/COVID-19: Empfehlungen zu Diagnostik und Therapie. Anästhesiologie [Internet]. 2020 [cited 2020 Mai 20];55(04):257-265. Available from: https://www.thieme-connect.de/products/ejournals/ html/10.1055/a-1146-8674.

14. Lingzhong Meng MD, Haibo Qiu MD, Li Wan MD, et al. Intubation and Ventilation amid the COVID-19 Outbreak: Wuhan's Experience. Anesthesiology [Internet]. 2020 [cited 2020 Mai 20]; 132(6):1317-1332. Available from: https://anesthesiology.pubs.asahq. org/solr/searchresults. aspx?author=Zhanggang+Xue.

15. Ong SWX, Tan YK, Chia PY, Lee TH, Ng OT, Wong MSY, et al. Air, surface environmental, and personal protective equipment contamination by severe acute respiratory syndrome coronavirus 2 (SARS-CoV-2) from a symptomatic patient. JAMA [Internet]. 2020 [cited 2020 Mai 20]; 323(16):1610-1612. Available from:: https://jamanetwork.com/journals/jama/fullarticle/2762692.

16. Crepaldi MA, Schmidt B, Noal DS, Bolze SDA, Gabarra LM. Terminalidade, morte e luto na pandemia de COVID-19: demandas psicológicas emergentes e implicações práticas. Estud. [Internet]. 2020 [cited 2020 Jul 24]; 37:e200090. Available from: http://dx. doi.org/10.1590/1982-0275202037e200090 